\title{
The role and mechanism of microRNA-18a-5p in oral squamous cell carcinoma
}

\author{
CHENYAO HUANG ${ }^{1}$, HONGNING SONG ${ }^{2}$ and LINFENG LAI ${ }^{1}$ \\ ${ }^{1}$ Department of Oral and Maxillofacial Surgery, Wenzhou Central Hospital, Wenzhou, Zhejiang 325000; \\ ${ }^{2}$ Department of Oral and Maxillofacial Surgery, Affiliated Hospital of Taishan Medical University, \\ Tai'an, Shandong 271000, P.R. China
}

Received October 5, 2018; Accepted March 29, 2019

DOI: $10.3892 / \mathrm{mmr} .2019 .10403$

\begin{abstract}
The purpose of this study was mainly to explore the role and mechanism of microRNA-18a-5p (miR-18a-5p) in oral squamous cell carcinoma (OSCC). The expression of miR-18a-5p in OSCC cells and normal cells was firstly detected using reverse transcription-quantitative polymerase chain reaction (RT-qPCR). The cell viability, apoptosis, migration and invasion abilities of OSCC cells were determined by MTT, cell apoptosis, wound healing and Transwell assays respectively. Additionally, bioinformatics software analysis and luciferase reporter assays were performed to predict and confirm the candidate target of miR-18a-5p. Western blot analysis was used to assess protein expression. It was revealed that the expression of miR-18a-5p in OSCC cells was higher than that in normal cells. In vitro studies revealed that the cell viability, migration and invasion abilities of OSCC cells were promoted and cell apoptosis was inhibited by miR-18a-5p overexpression. In addition, $\operatorname{Smad} 2$ was identified as a target of miR-18a-5p. It was also revealed that miR-18a-5p overexpression significantly inhibited the expression of Smad2, Smad4 and E-cadherin, and the levels of Smad7, collagen I, transforming growth factor- $\beta$ (TGF- $\beta$ ), $\alpha$-smooth muscle actin ( $\alpha$-SMA), vimentin were enhanced. While miR-18a-5p downregulation presented the opposite effects. In conclusion, the results indicated that $\mathrm{miR}-18 \mathrm{a}-5 \mathrm{p}$ can regulate the biological process of OSCC by targeting Smad2 and miR-18a-5p/Smad2 may be potential therapeutic targets for OSCC.
\end{abstract}

\section{Introduction}

Head and neck cancer, including oral squamous cell carcinoma (OSCC), is the sixth leading cancer worldwide (1). More than

Correspondence to: Dr Linfeng Lai, Department of Oral and Maxillofacial Surgery, Wenzhou Central Hospital, 32 Da Jian Lane, Jiang Bin West Road, Wenzhou, Zhejiang 325000, P.R. China E-mail: lailf1828@163.com

Key words: oral squamous cell carcinoma, miR-18a-5p, SMAD2, invasion, migration, apoptosis
400,000 people succumb to OSCC every year, and the 5-year survival rate for patients with OSCC is relatively poor $(2,3)$. Recently, medical technology for OSCC treatment has been greatly improved, and treatments of OSCC mainly include surgery, radiation or a combination of methods (4). However, more than half of OSCC patients are diagnosed in advanced stages. Due to the high recurrence rate and distant metastases in patients with advanced OSCC, it is worthwhile to improve current medical treatment approaches, and the exploration of the molecular mechanism in the development process of OSCC is greatly required.

MicroRNAs (miRNAs/miRs) are a class of small non-coding RNA molecules that function in RNA silencing and post-transcriptional regulation of gene expression (5). Due to their important role in the pathogenesis of cancer, miRNAs are regarded as biomarkers and therapeutic targets in various types of cancer. Researchers revealed that the aberrant expression of miR-18a-5p was associated with the occurrence and development of multiple malignant cancers, such as lung (6), esophageal (7) and gastric cancer (8). However, the role and mechanism of miR-18a-5p in OSCC are still unclear. It is now generally accepted that the transforming growth factor- $\beta$ (TGF- $\beta$ ) signaling pathway also plays an important role in the development of OSCC (9). However, the underlying mechanism concerning the involvement of TGF- $\beta$ in the progression of OSCC is not yet fully understood. Smad2 as a potent tumor-suppressive gene, is also an important component of TGF- $\beta$ signal transduction (10).

The purpose of the present study was to investigate the role of miR-18a-5p in OSCC cells in vitro, and to explore the molecular mechanism. We hope to provide more theoretical basis and treatment strategies for the diagnosis and treatment of OSCC.

\section{Materials and methods}

Cell culture and cell transfection. The OSCC cell line (SCC9) and the primary normal human oral keratinocyte (HOK) cells were originally acquired from the American Type Culture Collection (ATCC, Manassas, VA, USA). SCC9 cells were grown in RPMI-1640 medium containing $10 \%$ fetal bovine serum (FBS; both from Invitrogen; Thermo Fisher Scientific, Inc., Waltham, MA, USA) and $1 \%$ penicillin-streptomycin 
solution (Sigma-Aldrich; Merck KGaA, Darmstadt, Germany), and incubated in an incubator at $37^{\circ} \mathrm{C}$ with $5 \% \mathrm{CO}_{2}$. HOK cells were grown in RPMI-1640 medium containing $15 \%$ FBS, and incubated at $37^{\circ} \mathrm{C}$ with $5 \% \mathrm{CO}_{2}(11)$.

SCC 9 cells $\left(3 \times 10^{4}\right.$ cells/well) were transiently transfected with the negative control (NC) (forward: 5'-UUCUCCGAA CGUGUCACGUTT-3'; and reverse: 5'-ACGUGACACGUU CGGAGAATT-3'); the miR-18a-5p mimic (forward: 5'-UAA GGUGCAUCUAGUGCAGAUAG-3'; and reverse: 5'-AUC UGCACUAGAUGCACCUUAUU-3') or the miR-18a-5p inhibitor (5'-CUAUCUGACUAGAUGCACCUUA-3') respectively using Lipofectamine 2000 (Invitrogen; Thermo Fisher Scientific, Inc.) according to the manufacturer's instructions. Transfection efficiency was detected $48 \mathrm{~h}$ after the transfection experiment.

MTT assay. MTT assays were performed to assess cell viability. Logarithmic phase cells were seeded in a 96-well plate with $1 \times 10^{4}$ cells/well and incubated at $37^{\circ} \mathrm{C}$ in a $5 \% \mathrm{CO}_{2}$ incubator for 12,24 or $48 \mathrm{~h}$ respectively, after which $20 \mu \mathrm{l}$ of MTT solution $(5 \mathrm{mg} / \mathrm{ml}$ in distilled water) was added to each well, and cells were incubated for a further $2 \mathrm{~h}$ at $37^{\circ} \mathrm{C}$ with $5 \%$ $\mathrm{CO}_{2}$. The absorbance was assessed at a wavelength of $450 \mathrm{~nm}$ using a microplate reader.

Cell apoptosis assay. After specific treatment, SCC9 cells were collected and washed with cold PBS at least three times. SCC9 cell apoptosis was assessed by cell apoptosis assay. In brief, SCC9 cells $\left(1 \times 10^{6}\right)$ from various groups (control, NC, mimic and inhibitor) were firstly re-suspended in binding buffer, and then labeled with Annexin V-FITC and propidium iodide (PI) (BD Biosciences, San Diego, CA, USA) in line with the manufacturer's instructions. Flow cytometry (BD FACS Aria; BD Biosciences, Franklin Lakes, NJ, USA) was applied to analyze cell apoptosis. The experiment was repeated at least three times.

Transwell assay. In vitro invasion assays were performed using Transwell plates (BD Biosciences) with $8-\mu \mathrm{m}$ pores. The SCC9 cells ( $1 \times 10^{4}$ cells) in RPMI-1640 medium were added to the upper chamber of the Transwell plates. Then RPMI-1640 medium containing $20 \%$ FBS as a chemo-attractant was added to the lower chamber. After $48 \mathrm{~h}$ of incubation, cells on the upper surface were removed using cotton wool and the cells on the bottom surface of the membrane were fixed with methanol and stained with $0.5 \%$ crystal violet. Images were captured at an $\times 200$ magnification and the cells were counted using a light microscope (Olympus Corporation, Tokyo, Japan).

Wound-healing assay. For the wound-healing assay, confluent monolayers of SCC9 cells cultured in 24-well plates were mechanically wounded using a $10-\mu l$ pipette tip. The wells were washed to remove cellular debris and the cells were allowed to migrate for $48 \mathrm{~h}$. Representative images were captured at an x200 magnification under an inverted microscope (Olympus Corporation). The experiments were repeated at least three times. This assay was performed $48 \mathrm{~h}$ after transfection.

Reverse transcription-quantitative polymerase chain reaction (RT-qPCR). TRIzol ${ }^{\circledR}$ reagent (Invitrogen; Thermo Fisher
Scientific, Inc.) was used to extract the total RNA from the cells. GAPDH or U6 was used as an internal control for mRNA or miRNA expression. cDNAs were generated by using the PrimeScript ${ }^{\mathrm{TM}}$ RT reagent kit (Takara Bio, Inc.) in line with the manufacturer's instructions. SYBR Premix Ex Taq (Takara Bio, Inc.) was carried out to analyze the synthesized cDNAs according to the manufacturer's instructions. Primer sequences used for qPCR were obtained as required and listed as following: miR-18a-5p forward, 5'-ACGTAAGGTGCA TCTAGTGCAGATA-3' and reverse, 5'-GTGCAGGGTCCG AGGT-3'; $\alpha$-smooth muscle actin ( $\alpha$-SMA) forward, 5'-GTG TTGCCCCTGAAGAGCAT-3' and reverse, 5'-GCTGGGACA TTGAAAGTCTCA-3'; E-cadherin forward, 5'-CGAGAG CTACACGTTCACGG-3' and reverse, 5'-GGGTGTCGA GGGAAAAATAGG-3'; vimentin forward, 5'-GACGCCATC AACACCGAGTT-3' and reverse, 5'-CTTTGTCGTTGGTTA GCTGGT-3'; collagen I forward, 5'-GGCTTCCCTGGTCTT CCTGG-3' and reverse, 5'-CCAGGGGGTCCAGCCAAT-3'; TGF- $\beta$ forward, 5'-GTCCCTGAAGTCAGCTGCATA-3' and reverse, 5'-TGGGACAGTCCAGTTCTTCAT-3'; U6 forward, 5'-GCTTCGGCAGCACATATACTAAAAT-3' and reverse, 5'-CGCTTCACGAATTTGCGTGTCAT-3'; GAPDH forward, 5'-GGAGTCCACTGGCGTCTTCA-3' and reverse, 5'-GTC ATGAGTCCTTCCACGATACC-3'. The $2^{\Delta \Delta \mathrm{Cq}}$ method (12) was performed for the calculation of the relative expression of the genes.

Western blot analysis. After specific treatment, total cellular proteins from SCC9 cells were extracted using RIPA Buffer (Auragene, Changsha, China). A BCA protein quantitative kit (Thermo Fisher Scientific, Inc.) was used to assess the concentration of protein samples. An equal amount of protein samples (30 $\mu \mathrm{g} / \mathrm{lane})$ were resolved by $12 \%$ SDS-PAGE and then transferred onto PVDF membranes. The membranes were blocked with $5 \%$ non-fat milk at room temperature for $1 \mathrm{~h}$, followed by incubation with primary antibodies: Smad2 (cat. no. 5339; 1:1,000; Cell Signaling Technology, Inc., Danvers, MA, USA), Smad4 (cat. no. 46,535; 1:1,000; Cell Signaling Technology, Inc.), Smad7 (cat. no. ab90086; dilution: 1: 1,000; Abcam, Cambridge, MA, USA), collagen I (cat. no. ab34710; 1:1,000; Abcam), TGF- $\beta$ (cat. no. 3709; 1:1,000; Cell Signaling Technology, Inc.), $\alpha$-SMA (cat. no. 68,$463 ; 1: 1,000$; Cell Signaling Technology, Inc.), vimentin (cat. no. 12826; 1:1,000; Cell Signaling Technology, Inc.), E-cadherin (cat. no. 3195; 1:1,000; Cell Signaling Technology, Inc.), and $\beta$-actin (cat. no. 12620; 1:1,000; Cell Signaling Technology, Inc.) at $4^{\circ} \mathrm{C}$ overnight. Subsequently, the membranes were incubated with anti-rabbit immunoglobulin $\mathrm{G}$ horseradish peroxidase (HRP)-conjugated secondary antibodies (cat no. 7074; 1:2,000; Cell Signaling Technology, Inc.) at room temperature for $2 \mathrm{~h}$. Notably, secondary antibodies were not used when the primary antibodies were HRP-conjugated. To visualize the protein blots, an ECL kit (Applygen Technologies, Inc., Beijing, China) was used according the manufacturer's protocol. Densitometric semi-quantification was performed with ImageJ 1.38X software (National Institutes of Health, Bethesda, MD, USA).

Dual luciferase reporter assay. To predict the targets of miR-18a-5p, TargetScan bioinformatics software (www. 

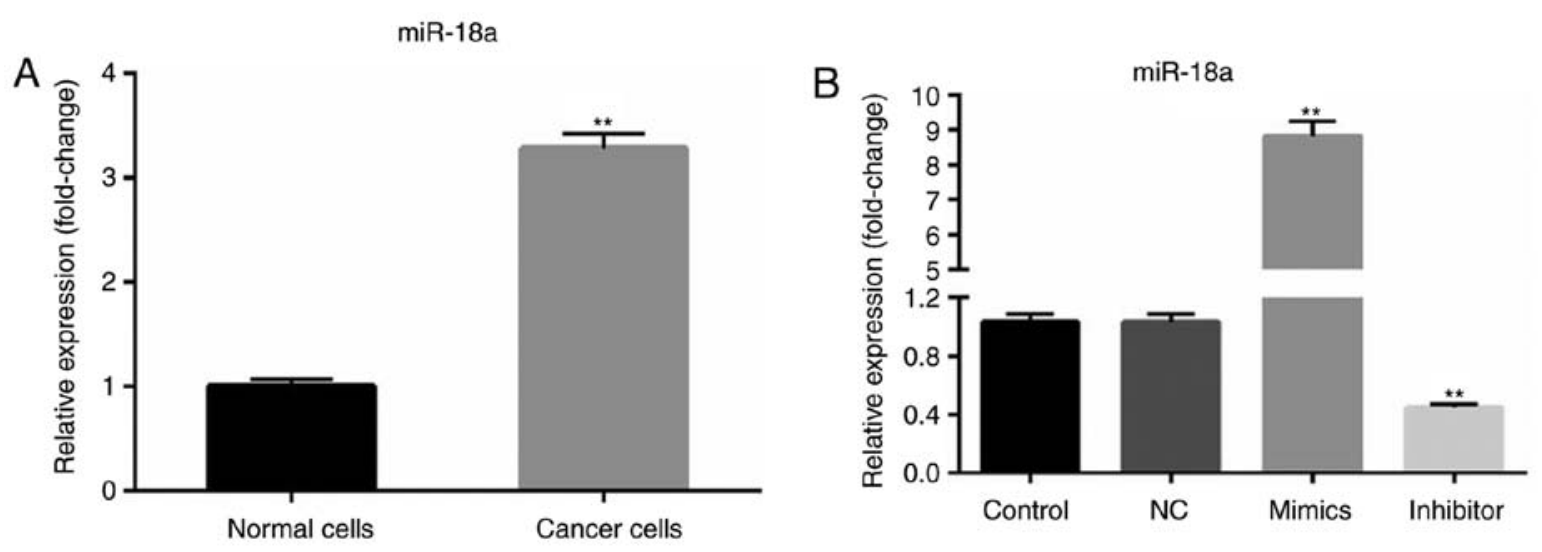

Figure 1. miR-18a-5p is upregulated in the oral squamous cell carcinoma cell line. (A) The relative expression level of miR-18a-5p was examined using RT-qPCR ("* $\mathrm{P}<0.01$ vs. normal cells). (B) A total of $48 \mathrm{~h}$ after transfection with miR-18a-5p mimics or the miR-18a-5p-inhibitor group, the level of miR-18a-5p was examined using RT-qPCR. ${ }^{* *} \mathrm{P}<0.01$ vs. the control. miR-18a-5p, microRNA-15a-5p; NC, negative control; RT-qPCR, reverse transcription-quantitative polymerase chain reaction.

targetscan.org/vert_71) was used, and results revealed that Smad2 was a potential target of miR-18a-5p. To confirm the direct binding sites, the wild-type (WT-Smad2) and mutant (MUT-Smad2) 3'-untranslated region (3'-UTR) of Smad2 were cloned into a pmiR-RB-Report ${ }^{\mathrm{TM}}$ dual luciferase reporter gene plasmid vector (Guangzhou RiboBio Co., Ltd., Guangzhou, China). To point-mutate the miR-18a-5p binding domain on the 3'UTR of Smad2, a QuikChange Site-Directed Mutagenesis Kit (Stratagene; Agilent Technologies, Inc., Santa Clara, CA, USA) was used as per the manufacturer's instructions. SCC9 cells were co-transfected with $100 \mathrm{ng}$ WT-Smad2 or $100 \mathrm{ng}$ MUT-Smad2 and $50 \mathrm{nM}$ miR-18a-5p (miR mimic) or $50 \mathrm{nM}$ negative control (NC) using Lipofectamine ${ }^{\circledR} 2000$ (Invitrogen; Thermo Fisher Scientific, Inc.) following the manufacturer's protocols. Luciferase activity was assessed, $48 \mathrm{~h}$ later, using the Dual-luciferase assay system (Promega Corporation, Madison, WI, USA) according to the manufacturer's protocol. Luciferase activity was normalized to Renilla luciferase activity.

Statistical analysis. Experiments were performed at least three times. All data were displayed as the mean \pm SD. SPSS 18.0 statistical software (SPSS, Chicago, IL, USA) was performed for statistical analyses. Comparisons between groups were performed by using Student's t-test or one-way analysis of variance followed by Tukey's post hoc test. $\mathrm{P}<0.05$ was considered to indicate a statistically significant difference.

\section{Results}

Upregulation of miR-18a-5p is revealed in OSCC cells. Using the RT-qPCR method, the expression level of miR-18a-5p in OSCC cells (SCC9 cell line) was determined. RT-qPCR analysis revealed that compared with primary normal human oral keratinocyte (HOK) cells, miR-18a-5p was upregulated in SCC9 cells (Fig. 1A). Then, SCC9 cells were transfected with the NC, miR-18a-5p mimics or miR-18a-5p inhibitor. It was revealed that miR-18a-5p was significantly upregulated in the miR-18a-5p mimic-transfected SCC9 cells and downregulated in miR-18a-5p inhibitor-transfected SCC9 cells (Fig. 1B).

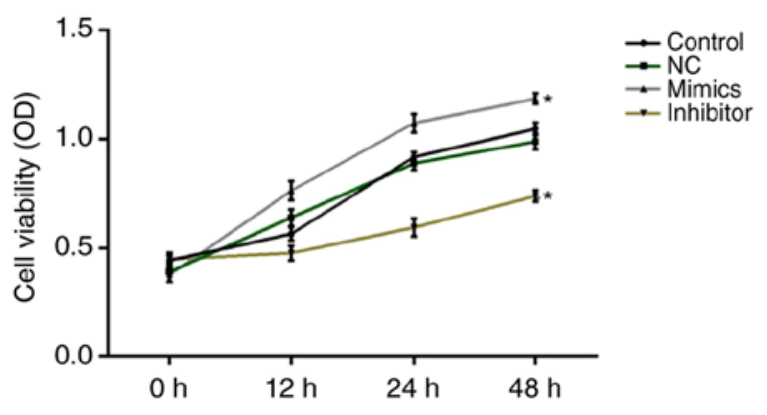

Figure 2. Effect of miR-18a-5p in SCC9 cell viability. A total of $48 \mathrm{~h}$ after transfection with miR-18a-5p mimics or the miR-18a-5p-inhibitor group, SCC9 cell viability was detected using MTT assays. ${ }^{*} \mathrm{P}<0.05$ vs. the control. miR-18a-5p, microRNA-15a-5p; NC, negative control; OD, optical density.

Effect of miR-18a-5p on the cell viability of SCC9 cells in vitro. At $0,12,24$ and $48 \mathrm{~h}$ after cell transfection, cell viability was assessed using MTT assays. The results revealed that miR-18a-5p mimics significantly increased SCC9 cell viability, while miR-18a-5p inhibitor significantly decreased SCC9 cell viability at all the time-points (Fig. 2).

Effect of miR-18a-5p on apoptosis of SCC 9 cells in vitro. Next, whether miR-18a-5p has an effect on apoptosis in SCC9 cells was determined by flow cytometry. The results revealed that the apoptosis rate of SCC9 cells in the miR-18a-5p inhibitor-transfection group was significantly upregulated compared with the control group. However, the rate of apoptosis in the miR-18a-5p mimic-transfection group was lower than that in the control group. Collectively, the results indicated that miR-18a-5p mimics inhibited the apoptosis of SCC9 cells in vitro, while the miR-18a-5p inhibitor significantly induced SCC9 cell apoptosis (Fig. 3).

Effect of miR-18a-5p on the invasion and migration of SCC9 cells in vitro. To assess the role of miR-18a-5p in the migration and invasion of OSCC cells, wound healing and Transwell assays were carried out. SCC9 cells were transfected with miR-18a-5p mimics or the inhibitor for $48 \mathrm{~h}$, and then wound healing and Transwell assays were performed. The wound 

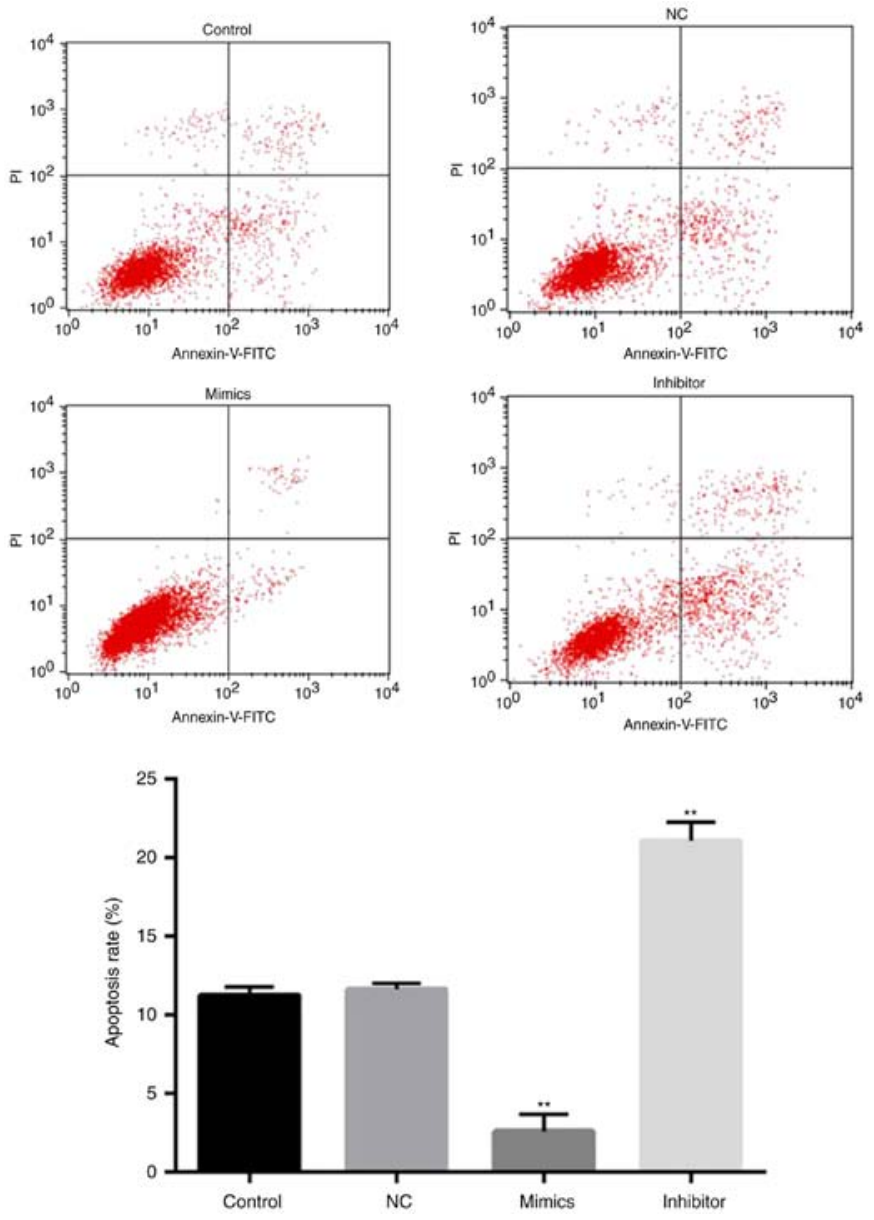

Figure 3. Effects of miR-18a-5p on SCC9 cell apoptosis. A total of $48 \mathrm{~h}$ after transfection with miR-18a-5p mimics or the miR-18a-5p-inhibitor group, flow cytometry was used to analyze cell apoptosis, and the apoptosis rate was calculated and presented. ${ }^{* *} \mathrm{P}<0.01$ vs. the control. miR-18a-5p, microRNA-15a-5p; $\mathrm{NC}$, negative control; PI, propidium iodide.
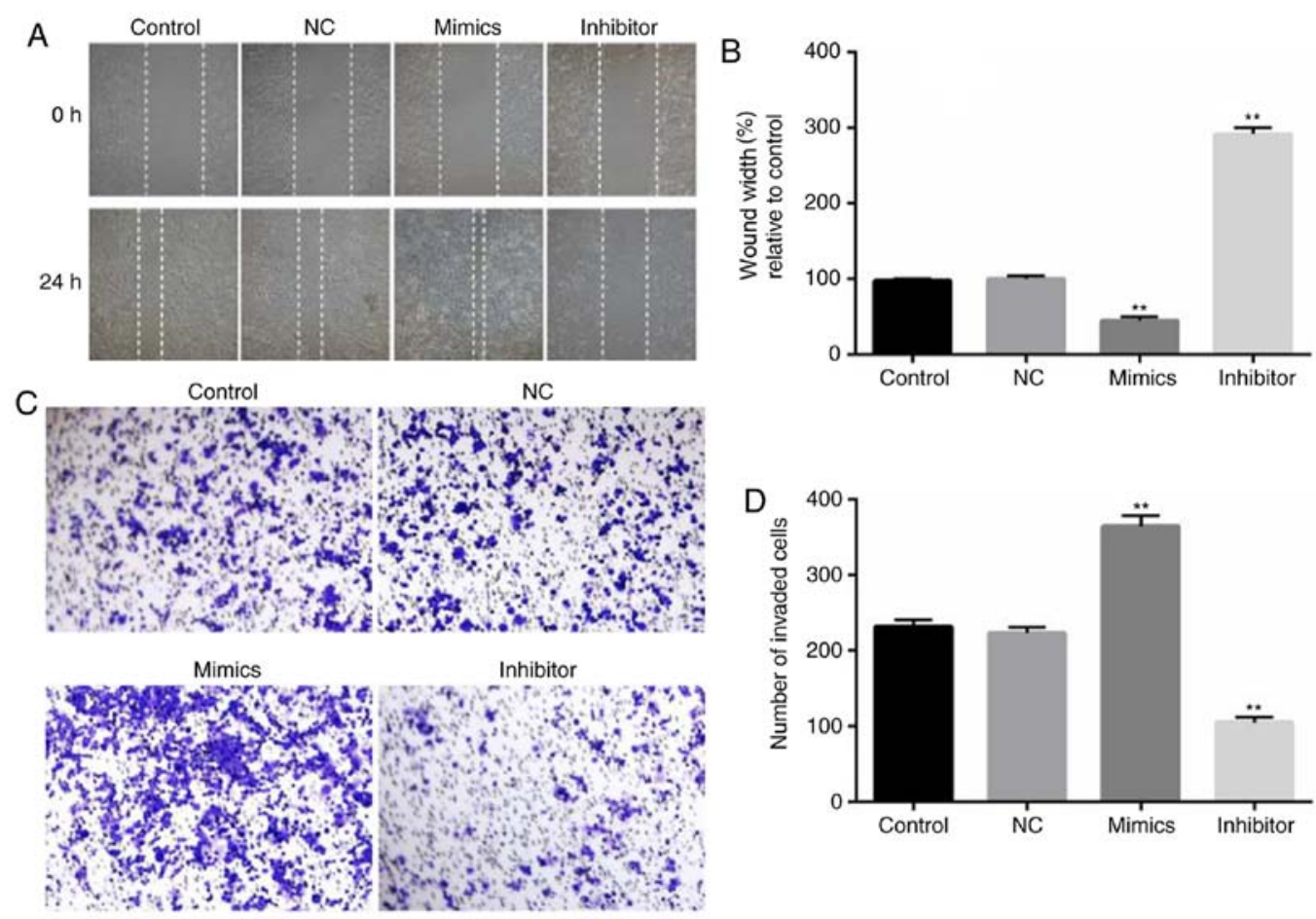

Figure 4. Effect of miR-18a-5p on the migration and invasion abilities of SCC9 cells. A total of $48 \mathrm{~h}$ after transfection with miR-18a-5p mimics or the miR-18a-5p-inhibitor group, (A and B) a wound healing assay (magnification, x100) was used to analyze cell migration, and (C and D) a Transwell assay (magnification, $\mathrm{x} 200$ ) was used to detect cell invasion. ${ }^{* *} \mathrm{P}<0.01$ vs. the control. miR-18a-5p, microRNA-15a-5p; NC, negative control. 
A Position 137-143 of SMAD2 3' UTR hsa-miR-18a-5p

Mutant position of SMAD2 3' UTR
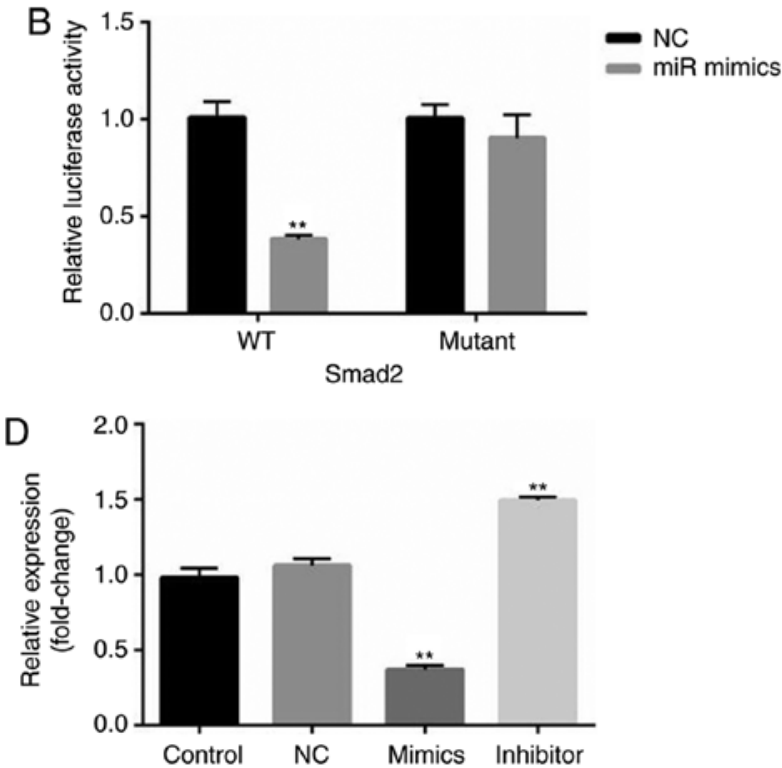

5 '... GUCUCAUCAAUUAAAGCACCUUG...

II $\|$ II II

5'... GUCUCAUCAAUUAAACGCGGAAG...
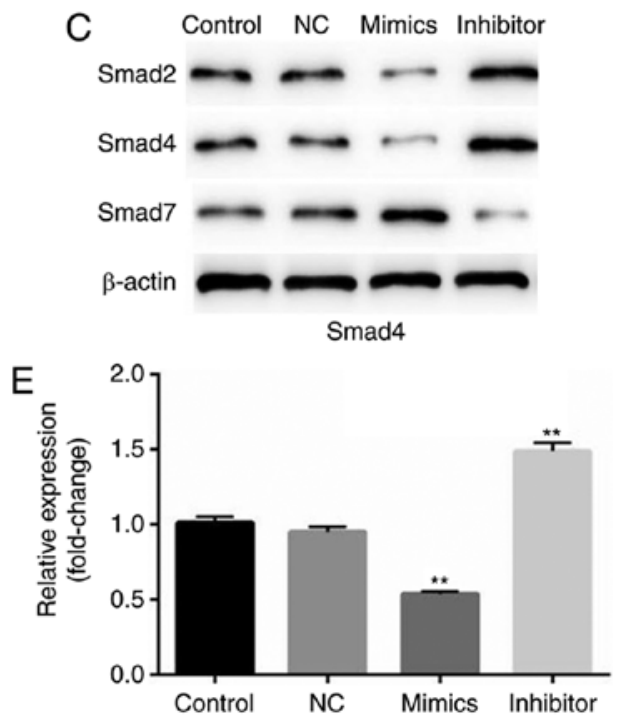

Smad7

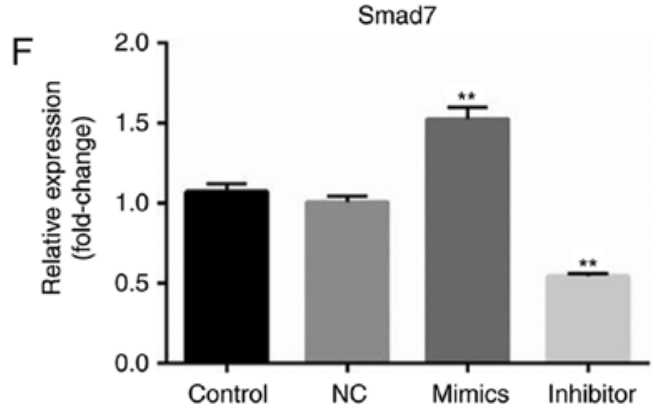

Figure 5. miR-18a-5p directly targets Smad2. (A) Binding sites between the 3'-UTR of Smad2 and miR-18a-5p. (B) A dual-luciferase reporter assay was used to detect the luciferase activity. ${ }^{* *} \mathrm{P}<0.01$ vs. NC. (C) Protein expression levels of Smad2, Smad4 and Smad7 were examined by western blotting in SCC9 cells. (D-F) mRNA levels of Smad2, Smad4 and Smad7 were assessed by reverse transcription-quantitative polymerase chain reaction. ${ }^{* *} \mathrm{P}<0.01$ vs. the control. 3'-UTR, 3'-untranslated region; miR-18a-5p, microRNA-15a-5p; NC, negative control.

healing assay revealed that compared with the control group, SCC9 cell migration ability significantly decreased in the miR-18a-5p inhibitor-transfection group, while the miR-18a-5p mimics significantly promoted the migration of SCC9 cells (Fig. 4A and B). As revealed in Fig. 4C and D, the number of invaded OSCC cells in the miR-18a-5p mimic-transfected group significantly increased, while it was decreased in the miR-18a-5p inhibitor-transfected group.

Smad2 is a target of $m i R-18 a-5 p$. According to the bioinformatics software analysis, it was observed that Smad2 was a potential target of miR-18a-5p (Fig. 5A). Thus, it was hypothesized that miR-18a-5p may play a role in OSCC cells by regulating $\mathrm{Smad} 2$. Therefore, a luciferase reporter assay was performed. As revealed in Fig. 5B, it was revealed that compared with the cells co-transfected with Smad2-WT and $\mathrm{NC}$, the relative luciferase activity significantly decreased in cells co-transfected with Smad2-WT and miR-18a-5p mimics. While no significant differences of the relative luciferase activity were revealed between the cells co-transfected with Smad2-MUT and NC, and cells co-transfected with Smad2-MUT and miR-18a-5p mimics.

In addition, the protein expression of Smad2, Smad4 and Smad7 was assessed in the present study. The results demonstrated that Smad2 and Smad4 protein expression in the miR-18a-5p mimic-transfected SCC9 cells was significantly decreased, while Smad7 expression was increased. The miR-18a-5p inhibitor had the opposite effects on the protein expression of Smad2, Smad4 and Smad7 in SCC9 cells. These results indicated that miR-18a-5p may play a role in the TGF- $\beta$ pathway.

The role of miR-18a-5p in the TGF- $\beta$ pathway. Finally, the biological function of the involvement of miR-18a-5p in the TGF- $\beta$ pathway was observed. After $48 \mathrm{~h}$ of transfection, the expression of collagen I, TGF- $\beta, \alpha$-SMA, vimentin, E-cadherin were assessed by western blotting method. Western blot analysis confirmed that overexpression of miR-18a-5p resulted in higher protein expression of collagen I, TGF- $\beta, \alpha$-SMA, vimentin and lower protein expression of E-cadherin. Additionally, the expression of collagen I, TGF- $\beta, \alpha$-SMA, and vimentin 


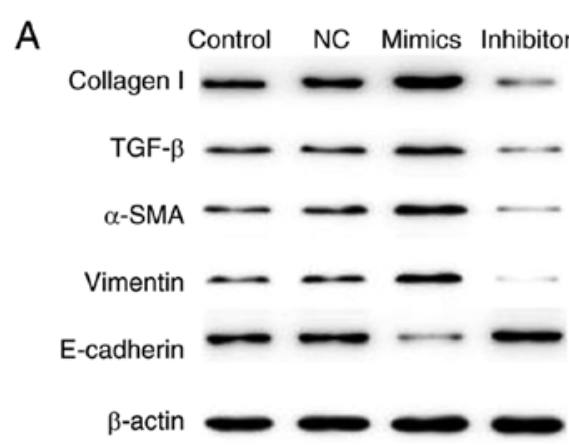

C
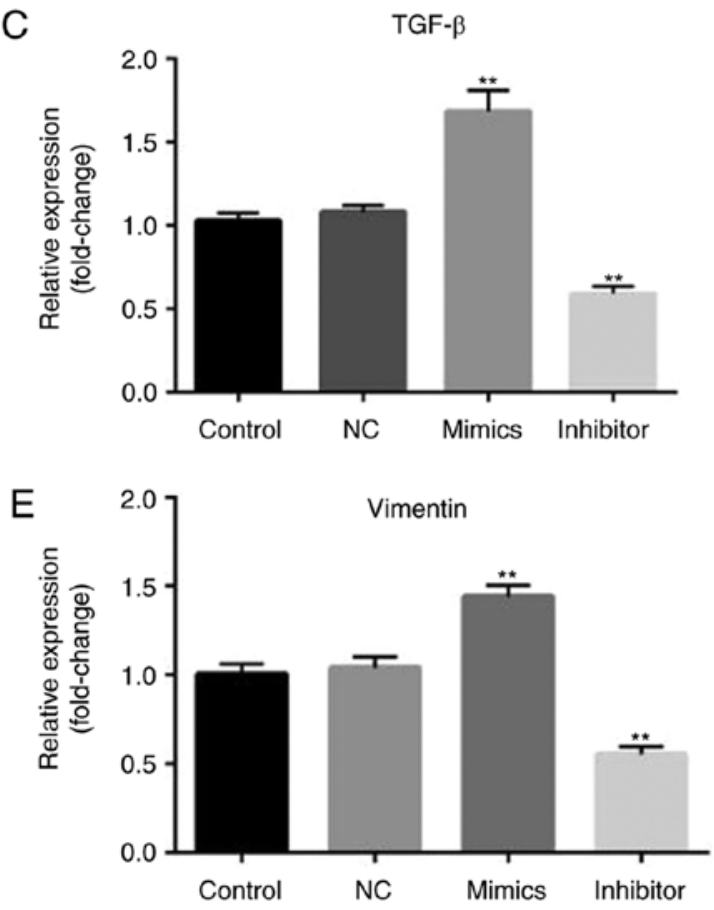

B
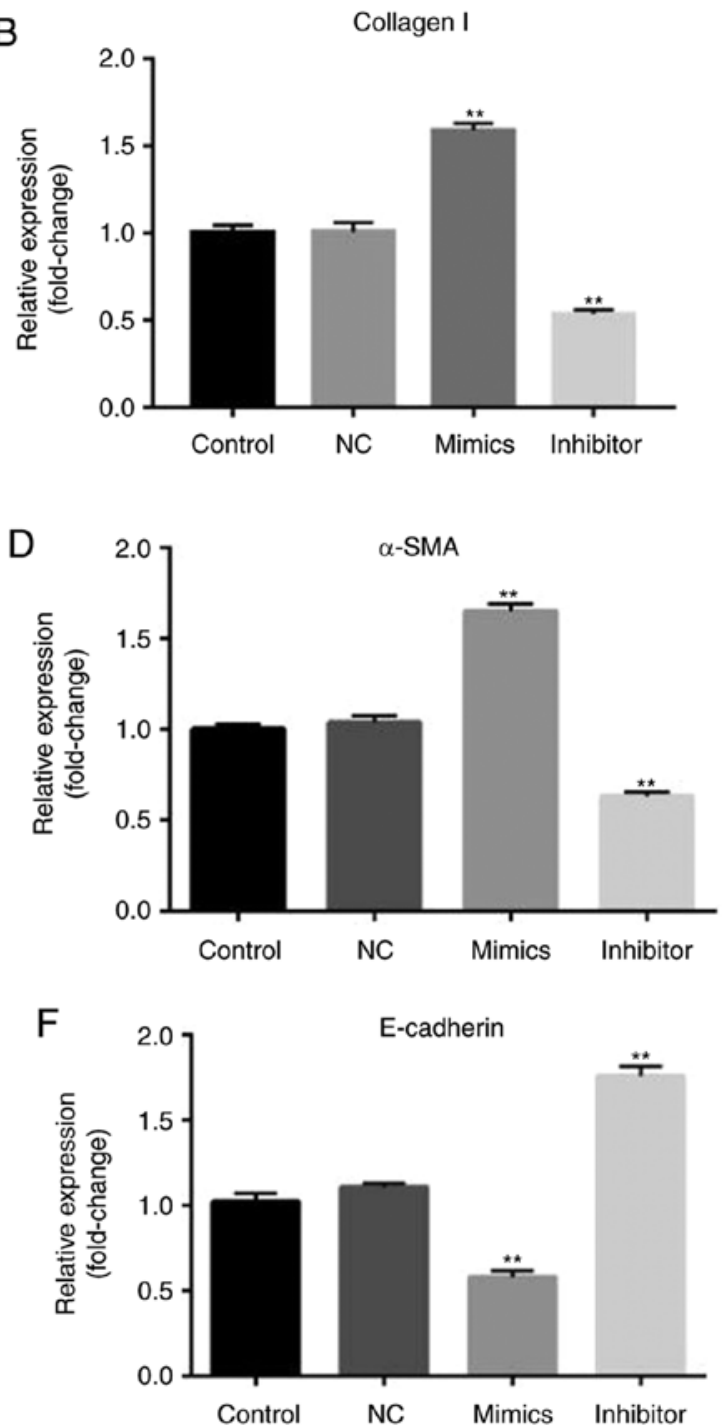

Figure 6. Effect of miR-18a-5p on the expression of collagen I, TGF- $\beta, \alpha$-SMA, vimentin, and E-cadherin. A total of $48 \mathrm{~h}$ after transfection with miR-18a-5p mimics or the miR-18a-5p-inhibitor group, (A) the protein level of collagen I, TGF- $\beta, \alpha$-SMA, vimentin, E-cadherin expression levels was assessed using a western blot assay, and (B-F) the mRNA level of collagen I, TGF- $\beta, \alpha$-SMA, vimentin and E-cadherin was detected using reverse transcription-quantitative polymerase chain reaction. ${ }^{* *} \mathrm{P}<0.01$ vs. the control. $\alpha$-SMA, $\alpha$-smooth muscle actin, miR-18a-5p, microRNA-15a-5p; NC, negative control; TGF- $\beta$, transforming growth factor- $\beta$.

was reduced and E-cadherin expression was enhanced in the miR-18a-5p inhibitor-transfected SCC9 cells (Fig. 6).

\section{Discussion}

Due to its poor prognosis and low survival rate, OSCC has become a global health problem (13). Accumulating evidence has demonstrated the important biological function of miRNAs in OSCC, however the underlying mechanism of miRNAs involved in the process of cancer is still poorly understood. The present study aimed to identify whether miR-18a-5p has an effect on the malignant biological behaviors of OSCC cells, and to explore the molecular mechanism.

Previous studies have identified the higher expression of miR-18a-5p in esophageal, colon, pancreatic and liver cancer as well as renal cell carcinoma. In addition, miR-18a-5p has been identified to play an important role in cancer cell proliferation, apoptosis, migration and invasion (7). In the present study, it was revealed that miR-18a-5p was highly expressed in SCC9 cells compared with normal cells. Then the effect of miR-18a-5p upregulation and miR-18a-5p downregulation was investigated on SCC9 cell viability, migration, invasion and apoptosis. The findings demonstrated that overexpression of miR-18a-5p could promote SCC9 cell viability, migration, invasion and inhibit cell apoptosis. While miR-18a-5p downregulation inhibited SCC9 cell viability, migration, invasion and induced cell apoptosis. These results indicated that miR-18a-5p acts as an oncogene to participate in OSCC progression and tumorigenesis.

It was further revealed that $\operatorname{Smad} 2$ was the potential target of miR-18a-5p, and in addition, it was determined that miR-18a-5p negatively regulated the expression of Smad2 and Smad4, and positively regulated the expression of Smad7. Studies have confirmed that Smad2 and Smad4 are two major downstream regulators in the TGF- $\beta 1 /$ Smad signaling pathway and both of them act as tumor suppressors in many human cancers, while Smad7 serves as a negative feedback regulator of this pathway $(14,15)$. Numerous studies have demonstrated 
that the TGF- $\beta 1 /$ Smad signaling pathway is an important pathogenic mechanism in various cancers, such as breast (16), prostate (17), and gastric cancer (18), as well as hepatocellular carcinoma (19) and esophageal squamous cell carcinoma (14). Thus, we speculated that miR-18a-5p may promote OSCC cancer cell biological progression though activation of the TGF- $\beta 1 /$ Smad signaling pathway and it may be an important therapeutic strategy for OSCC. Furthermore, our results indicated that miR-18a-5p could increase the expression of collagen I, $\alpha$-SMA and vimentin, but decrease E-cadherin expression. While miR-18a-5p inhibitor presented the opposite effects. It is well recognized, that collagen I as one of extracellular matrix components, plays an important role cancer cell infiltration and metastasis (20). $\alpha$-SMA has been revealed to be a negative prognostic marker and associated with cancer metastases (21). Vimentin is now being perceived as a canonical prognostic marker and has been revealed to have a high expression level in many cancers (22). Previous studies have demonstrated that E-cadherin, a well-known tumor suppressor protein (23), is involved in the TGF- $\beta 1 /$ Smad signaling pathway (24). All these findings were consistent with our present study. Thus, our findings indicated that high expression of miR-18a-5p may indicate a poor prognosis.

In conclusion, it was demonstrated that miR-18a-5p upregulation promoted cell viability, migration and invasion of OSCC cells and inhibited cell apoptosis in vitro by activating the TGF- $\beta 1 /$ Smad2 pathway. While miR-18a-5p downregulation presented the opposite effects. These data indicated that miR-18a-5p may be a promising therapeutic target for OSCC. However, this study is only a preliminary study of the role of miR-18a-5p in OSCC, and there are some limitations in our present study. In order to elucidate the role of miR-18a-5p in OSCC, numerous experiments are still required. For instance, the effect of miR-18a-5p on apoptosis-related proteins, such as caspases, should be determined. The level of miR-18a-5p in OSCC patients and the relationship between the level of miR-18a-5p in OSCC patients with the clinical characteristics of the patients should be clarified. In addition, in vivo studies should be performed to reveal the effect of miR-18a-5p on OSCC progression. These issues will be addressed in the future.

\section{Acknowledgements}

Not applicable.

\section{Funding}

No funding was received.

\section{Availability of data and materials}

The datasets used and/or analyzed during the present study are available from the corresponding author on reasonable request.

\section{Authors' contributions}

$\mathrm{CH}$ designed the present study, collected and analyzed the data, performed statistical analysis, searched the literature and prepared the manuscript. HS and LL performed statistical analysis and interpreted the data.

\section{Ethics approval and consent to participate}

Not applicable.

\section{Patient consent for publication}

Not applicable.

\section{Competing interests}

The authors declare that they have no competing interests.

\section{References}

1. Torre LA, Bray F, Siegel RL, Ferlay J, Lortet-Tieulent J and Jemal A: Global cancer statistics, 2012. CA Cancer J Clin 65: 87-108, 2015.

2. Kim JW, Park Y, Roh JL, Cho KJ, Choi SH, Nam SY and Kim SY: Prognostic value of glucosylceramide synthase and P-glycoprotein expression in oral cavity cancer. Int J Clin Oncol 21: 883-889, 2016.

3. Su CC, Lee KI, Chen MK, Kuo CY, Tang CH and Liu SH: Cantharidin induced oral squamous cell carcinoma cell apoptosis via the JNK-regulated mitochondria and endoplasmic reticulum stress-related signaling pathways. PLoS One 11: e0168095, 2016.

4. Lai YH, Liu H, Chiang WF, Chen TW, Chu LJ, Yu JS, Chen SJ, Chen HC and Tan BC: MiR-31-5p-ACOX1 axis enhances tumorigenic fitness in oral squamous cell carcinoma via the promigratory prostaglandin E2. Theranostics 8: 486-504, 2018.

5. Alsaweed M, Hartmann PE, Geddes DT and Kakulas F: MicroRNAs in breastmilk and the lactating breast: Potential immunoprotectors and developmental regulators for the infant and the mother. Int J Environ Res Public Health 12: 13981-14020, 2015.

6. Chen X, Wu L, Li D, Xu Y, Zhang L, Niu K, Kong R, Gu J, Xu Z, Chen $Z$ and Sun J: Radiosensitizing effects of miR-18a-5p on lung cancer stem-like cells via downregulating both ATM and HIF1 $\alpha$. Cancer Med 7: 3834-3847, 2018.

7. Zhou L, Li Z, Pan X, Lai Y, Quan J, Zhao L, Xu J, Xu W, Guan X, $\mathrm{Li} \mathrm{H}$, et al: Identification of miR-18a-5p as an oncogene and prognostic biomarker in RCC. Am J Transl Res 10: 1874-1886, 2018.

8. Li X,Luo F,LiQ, Xu M,Feng D, Zhang G and Wu W: Identification of new aberrantly expressed miRNAs in intestinal-type gastric cancer and its clinical significance. Oncol Rep 26: 1431-1439, 2011.

9. Hu L, Liu J, Li Z, Wang C and Nawshad A: Transforming growth factor- $\beta 1$ activates $\Delta \mathrm{Np} 63 / \mathrm{c}-\mathrm{Myc}$ to promote oral squamous cell carcinoma. Oral Surg Oral Med Oral Pathol Oral Radiol 122: 460-482.e4, 2016.

10. Heldin $\mathrm{CH}$ and Moustakas A: Signaling receptors for TGF- $\beta$ family members. Cold Spring Harb Perspect Biol 8: pii: a022053, 2016.

11. Zhou XL, Chen G, Li MX, Wang HX, Hong JW, Shen JY, Wang Q, Ge X, Ding Z and Xu LC: Targeting YOD1 by RNA interference inhibits proliferation and migration of Human Oral Keratinocytes through transforming growth Factor- $\beta 3$ signaling pathway. Biomed Res Int 2018: 6254308, 2018.

12. Livak KJ and Schmittgen TD: Analysis of relative gene expression data using real-time quantitative PCR and the 2(-Delta Delta C(T)) method. Methods 25: 402-408, 2001.

13. Liu L, Jiang H, Zhao J and Wen H: MiRNA-16 inhibited oral squamous carcinoma tumor growth in vitro and in vivo via suppressing Wnt $/ \beta$-catenin signaling pathway. Onco Targets Ther 11: 5111-5119, 2018.

14. Abudureheman A, Ainiwaer J, Hou Z, Niyaz M, Turghun A, Hasim A, Zhang H, Lu X and Sheyhidin I: High MLL2 expression predicts poor prognosis and promotes tumor progression by inducing EMT in esophageal squamous cell carcinoma. J Cancer Res Clin Oncol 144: 1025-1035, 2018.

15. Ullah I, Liao Y, Wan R, Tang L and Feng J: Alternative splicing of SMAD4 and its function in HaCaT cells in response to UVB irradiation. J Cancer 9: 3177-3186, 2018.

16. Yang R, Liang J, Xu GX, Ding LM, Huang HM, Su QZ, Yan J and Li YC: Human cytomegalovirus glycoprotein B inhibits migration of breast cancer MDA-MB-231 cells and impairs TGF- $\beta /$ Smad2/3 expression. Oncol Lett 15: 7730-7738, 2018. 
17. Kardooni H, Gonzalez-Gualda E, Stylianakis E, Saffaran S, Waxman J and Kypta RM: CRISPR-mediated reactivation of DKK3 expression attenuates TGF- $\beta$ signaling in prostate cancer. Cancers 10: pii: E165, 2018.

18. Peng J, Chen W, Chen J, Yuan Y,Zhang J and He Y: Overexpression of chloride channel-3 predicts unfavorable prognosis and promotes cellular invasion in gastric cancer. Cancer Manag Res 10: 1163-1175, 2018

19. Zhang T, Liu W, Meng W, Zhao H, Yang Q, Gu SJ, Xiao CC, Jia CC and Fu BS: Downregulation of miR-542-3p promotes cancer metastasis through activating TGF- $3 /$ Smad signaling in hepatocellular carcinoma. Onco Targets Ther 11: 1929-1939, 2018.

20. Su CQ, Qiu H and Zhang Y: Localization of keratin mRNA and collagen I mRNA in gastric cancer by in situ hybridization and hybridization electron microscopy. World J Gastroenterol 5: 527-530, 1999.

21. Sinn M, Denkert C, Striefler JK, Pelzer U, Stieler JM, Bahra M, Lohneis P, Dörken B, Oettle H, Riess H and Sinn BV: $\alpha$-Smooth muscle actin expression and desmoplastic stromal reaction in pancreatic cancer: Results from the CONKO-001 study. Br J Cancer 111: 1917-1923, 2014.
22. Dmello C, Sawant S, Alam H, Gangadaran P, Mogre S, Tiwari R, D'Souza Z, Narkar M, Thorat R, Patil K, et al: Vimentin regulates differentiation switch via modulation of keratin 14 levels and their expression together correlates with poor prognosis in oral cancer patients. PLoS One 12: e0172559, 2017.

23. Petrova YI, Schecterson L and Gumbiner BM: Roles for E-cadherin cell surface regulation in cancer. Mol Biol Cell 27: 3233-3244, 2016

24. Liang X, Zeng J, Wang L, Shen L, Ma X, Li S, Wu Y, Ma L, Ci X, Guo Q, et al: Histone demethylase RBP2 promotes malignant progression of gastric cancer through TGF- $31-(\mathrm{p}-\mathrm{Smad} 3)-\mathrm{RBP} 2-\mathrm{E}-$ cadherin-Smad3 feedback circuit. Oncotarget 6: 17661-17674, 2015.

This work is licensed under a Creative Commons Attribution-NonCommercial-NoDerivatives 4.0 International (CC BY-NC-ND 4.0) License. 